\title{
Proposed test for realist theories using Rydberg atoms coupled to a high- $Q$ resonator
}

\author{
S. F. Huelga, ${ }^{1}$ T. W. Marshall, ${ }^{*}$ and E. Santos ${ }^{2}$ \\ ${ }^{1}$ Departamento de Física, Universidad de Oviedo, 33007 Oviedo, Spain \\ ${ }^{2}$ Departamento de Física Moderna, Universidad de Cantabria, 39005 Santander, Spain
}

(Received 3 April 1995)

\begin{abstract}
From the hypotheses of realism and stationarity, the latter related to the Leggett-Garg assumption of noninvasive measurability, we derive inequalities, involving three autocorrelation functions, to be fulfilled by any two-state stochastic process. We seek to test a broad class of realist theories against quantum mechanics for a system consisting of a Rydberg atom interacting with a single quantized mode in a superconducting resonant cavity. Departures from quantum predictions are enhanced when the temperature is decreased to about $0.5 \mathrm{~K}$ and the state of the cavity approximates a Fock state.
\end{abstract}

PACS number(s): 03.65.Bz

Recent advances in optics have renewed interest in demonstrating the strange properties of quantum mechanics; for instance, those exemplified by the old Einstein-PodolskyRosen (EPR) [1] and Schrödinger's cat [2] paradoxes. Bell was able to refine the EPR paradox introducing (Bell) inequalities able to discriminate between quantum mechanics and local realism [3-5]. Following his work, many empirical tests of Bell inequalities have been performed in the past few years. In a similar way, the Schrödinger-cat paradox may be formalized by introducing inequalities able to test the existence of superpositions-distinguishable from mixtures-of macroscopic states. Inequalities of this kind were derived by Leggett and Garg [6] from the hypothesis of macroscopic realism and noninvasive measurability. Some empirical tests of these inequalities have been proposed [6-8], but no violation has been reported due to the difficulty of the experiments with truly macroscopic systems.

The aim of this paper is twofold. First we develop arguments to justify inequalities, similar to those obtained in [6], for the case of a system with mesoscopic dimensions, namely a Rydberg atom interacting with a tuned microwave cavity. Experiments using Rydberg atoms in microwave cavities have been proposed also for the test of Bell inequalities $[9,10]$. Apart from the novelty of testing Leggett-Garg-type inequalities, we think that our proposal is more feasible. In fact, Refs. $[9,10]$ consider highly idealized situations (e.g., absolute zero temperature, infinite $Q$ for the cavity, perfect angular correlation for the outgoing atoms, etc.). In contrast, we take into account explicitly all nonidealities and compare our proposal with experiments actually performed (see Figs. 2 and 3). The point is that nonidealities are usually enough to prevent an uncontroversial violation of Bell-type inequalities [11].

Consider a general two-state system. According to a realistic description, such a system can actually be in only one of these states, which we shall denote as $e$ (for "excited") and $g$ ( for "ground"); the notion of the superposition of $e$ and $g$, though useful as an algorithm for the calculation of probabilities, cannot represent a real state of the system. The

\footnotetext{
*Permanent address: Department of Mathematics, University of Manchester, Manchester M13 9PL, United Kingdom.
}

temporal evolution consists of random transitions between these two states. Let us now define the random variable

$$
\chi(t)= \begin{cases}+1, & \text { if the system is in state } e \text { at time } t, \\ -1, & \text { if the system is in state } g \text { at time } t .\end{cases}
$$

This variable has a definite value at all times $t$. The autocorrelation $K\left(t_{1}, t_{2}\right)$ of the process is defined as

$$
K\left(t_{1}, t_{2}\right)=\left\langle\chi\left(t_{1}\right) \chi\left(t_{2}\right)\right\rangle .
$$

As in Ref. [6], realism enters through the assumptions that

(i) Dynamical variables possess definite values at all instants.

(ii) A measurement of the state of the atom ( $e$ or $g$ ) at the exit of the cavity reveals nothing more than the state of the atom really existing before the measurement. That is, the measurement simply reveals the preexisting values without any change.

Consider now three different times $t_{i}(i=1,2,3)$. We define the joint probability

$$
P_{e e e}\left(t_{1}, t_{2}, t_{3}\right)=\operatorname{Prob}\left[\chi\left(t_{1}\right)=\chi\left(t_{2}\right)=\chi\left(t_{3}\right)=+1\right] .
$$

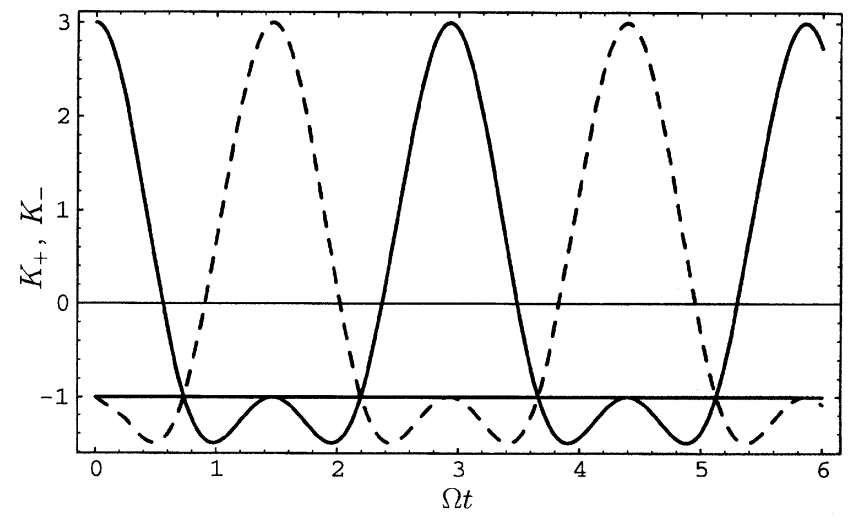

FIG. 1. Functions $K_{+}$and $K_{-}$(dashed) versus $\Omega t$ for the ideal case $T=0$. Violations of Leggett-Garg-type inequalities occur whenever either curve goes below -1 . 


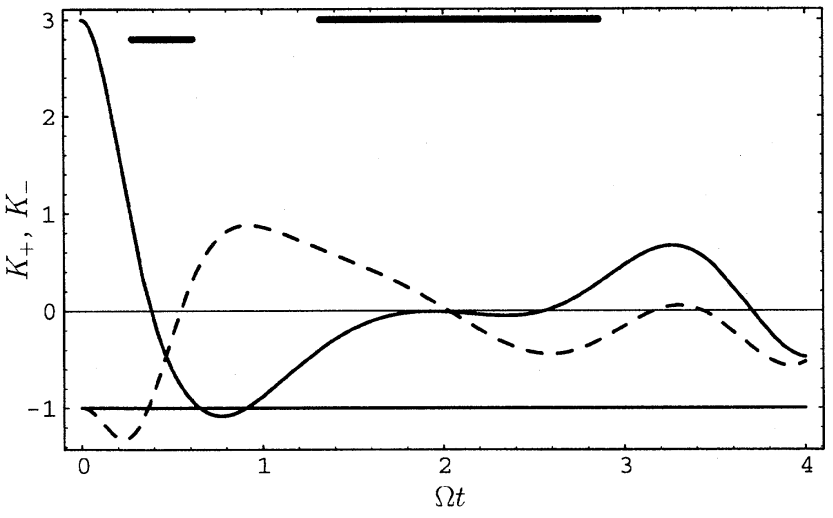

FIG. 2. $K_{ \pm}$versus $\Omega t$ at $T=3 \mathrm{~K}$. Violations of Leggett-Gargtype inequalities occur whenever either curve goes below -1 . The thick horizontal segments indicate the ranges of interaction times currently accessible for the two Rydberg transitions.

Another seven probabilities can be defined in a similar way; for example,

$$
P_{\text {ege }}\left(t_{1}, t_{2}, t_{3}\right)=\operatorname{Prob}\left[\chi\left(t_{1}\right)=\chi\left(t_{3}\right)=+1, \chi\left(t_{2}\right)=-1\right],
$$

and so on. In terms of these quantities we can construct autocorrelations for the three different time intervals as

$$
\begin{aligned}
K\left(t_{1}, t_{2}\right)= & P_{e e e}+P_{e e g}+P_{g g e}+P_{g g g}-P_{e g e}-P_{e g g}-P_{g e e} \\
& -P_{g e g}, \\
K\left(t_{2}, t_{3}\right)= & P_{e e e}-P_{e e g}-P_{g g e}+P_{g g g}-P_{e g e}+P_{e g g}+P_{g e e} \\
& -P_{g e g}, \\
K\left(t_{1}, t_{3}\right)= & P_{e e e}-P_{e e g}-P_{g g e}+P_{g g g}+P_{e g e}-P_{e g g}-P_{g e e} \\
& +P_{g e g} .
\end{aligned}
$$

Now, adding these three relations, we obtain

$$
K\left(t_{1}, t_{2}\right)+K\left(t_{2}, t_{3}\right)+K\left(t_{1}, t_{3}\right) \geqslant-1,
$$

whereas the combination (7)-(5)-(6) gives

$$
K\left(t_{1}, t_{3}\right)-K\left(t_{1}, t_{2}\right)-K\left(t_{2}, t_{3}\right) \geqslant-1 .
$$

These three-time inequalities are closely related to the fourtime inequalities of [6]. We shall see that, once the stationarity hypothesis has been introduced, they actually impose stronger constraints than do the four-time inequalities on the autocorrelation of the process.

In the Rydberg-atom version of a two-state system $[12,13]$, a set of atoms, prepared in the state $e$, interacts for a finite time with a microwave cavity tuned to the $e \rightarrow g$ transition frequency. The atoms emerge from the cavity in one of two states $(e, g)$. We may consider these as "pointer states," thereby emphasizing that they represent the (exhaustive and mutually exclusive) outcomes when the atom interacts with a certain two-channel detection apparatus. A realist (that is, hidden-variables) representation of such a state is an ensemble of microstates with always the same probability distribution of hidden variables. Thus, for example, an atom emerging in the state $e$ has precisely the same distribution of microstates as has the incident atom, and this state is associated with a definite state of the cavity, which also coincides with its initial state. This latter state also is associated with a definite distribution of microstates, and in this case we may identify the hidden variables as, for example, the amplitudes and phases of all the cavity modes. The same argument may be used to support the idea that an atom emerging in state $g$ is associated with a definite state of the cavity, which may be informally described as "the initial state plus one photon."

Equations (8) and (9), derived from realism alone, are compatible with quantum mechanics. This may be easily seen, for instance, in the example considered by Hardy et al. [14]. We shall introduce the additional assumption of stationarity. Supposing the time ordering $t_{1}<t_{2}<t_{3}$, the atom passes from its initial $e$ state at $t_{1}$, via $e$ or $g$ at $t_{2}$, to $e$ or $g$ at $t_{3}$. Given the above realist descriptions of $e$ and $g$, it seems plausible (though nothing more) that the evolution from $t_{1}$ to $t_{2}$ is governed by the same stochastic differential equation as the evolution from $t_{2}$ to $t_{3}$, and this implies stationarity; that is,

$$
K\left(t_{1}, t_{2}\right)=K\left(t_{1}-t_{2}\right) .
$$

There is a connection between this assumed stationarity and the Leggett-Garg assumption of noninvasive measurability, since the above description supposes that we could (counterfactually) intervene at any moment when the atom is inside the cavity, by removing it and observing in which of the two possible states it is. The supposition that each of these states corresponds to a definite state of the cavity, and that the atom's removal from the cavity means that an observation of its state cannot change the state of the cavity, is what Leggett and Garg called noninvasive measurability, and it contradicts the quantum property known as entanglement. This latter property becomes rather exotic for a macroscopic object but it is a direct consequence of the superposition of $|e, n\rangle$ and $|g, n+1\rangle$ [see Eq. (11) below]. Our stationarity assumption (10) may be considered as the formal statement of the condition of noninvasive measurability.

We therefore suggest that it is worthwhile to compare the predictions of a restricted family of realist processes, namely the stationary family, with the predictions of quantummechanics (QM) for this system. This comparison seems particularly attractive, if we take into account that the pure Jaynes-Cummings evolution of the atom-plus-cavity system (for further discussion see below) also takes a form that suggests stationarity, namely

$$
\begin{aligned}
|e, n\rangle \rightarrow & \cos \left[\Omega \sqrt{n+1}\left(t_{1}-t_{2}\right)\right]|e, n\rangle \\
& +\sin \left[\Omega \sqrt{n+1}\left(t_{1}-t_{2}\right)\right]|g, n+1\rangle .
\end{aligned}
$$

This latter property carries over to the more general case of the "stationary cavity"- a concept that we explain below.

Now, with the assumption of stationarity, consider the case

$$
t_{2}-t_{1}=t_{3}-t_{2}=t \text {. }
$$

Then the inequalities (8) and (9) become

$$
K_{+}(t)=K(2 t)+2 K(t) \geqslant-1,
$$




$$
K_{-}(t)=K(2 t)-2 K(t) \geqslant-1 \text {. }
$$

These inequalities impose a constraint on the statistical predictions of any realist theory of the family described above, and are amenable to an experimental test. With this aim, we propose to consider, as suitable two-level systems, atoms initially prepared in a highly excited (Rydberg) state interacting with a single mode in a superconducting cavity resonant with a selected Rydberg transition. At first sight it may seem surprising that a scenario previously used for testing features considered purely quantum mechanical; for example, the revivals of the Rabi oscillations predicted by the Jaynes-Cummings model [12] and the observation of subPoissonian photon statistics [13], could throw some light on the question posed here. However, two reasons may be advanced to justify this proposal:

(i) Due to their high principal quantum number ( $n \sim 60$ ), and since the relative energy differences between adjacent levels are small compared to the large changes between lower levels, Rydberg atoms are expected to show a number of classical properties.

(ii) The linear dimensions of these atoms are comparable with the size of large biomolecules; hence the use of the words mesoscopic dimensions. It should be noted that the word mesoscopic is used in cavity QED with a different meaning, namely defining a system with a large number of atoms and/or photons.

As is well known, the dipole interaction of a two-level atom with a single quantized mode of the electromagnetic field is described, in the rotating-wave approximation, by the Jaynes-Cummings Hamiltonian [15]. Provided that the atom is initially in its upper state, and that the cavity contains $n$ photons, the probability for an atom initially excited to remain in the excited state at time $t>0$ is given by

$$
P_{e e}(t ; n)=\cos ^{2}[\Omega \sqrt{n+1} t]
$$

where exact resonance is assumed. $\Omega$ is the single-photon Rabi frequency, which sets the time scale of the evolution. The autocorrelation $K(t)$ can be calculated as

$$
K(t)=p_{e}\left(P_{e e}-P_{e g}\right)+p_{g}\left(P_{g g}-P_{g e}\right)
$$

where $p_{e}$ and $p_{g}$ are the weight factors required for a stationary description, which coincide with the weights that are established after a long time (the relaxation time of the cavity) if ergodicity is assumed. From (15) and taking into account that $P_{g g}=P_{e e}$ and $P_{e g}=P_{g e}=1-P_{e e}$, the quantum autocorrelation becomes

$$
K_{Q}(t)=\cos [2 \Omega \sqrt{n+1} t]
$$

The behavior of the functions $K_{+}(t)$ and $K_{-}(t)$ according to QM has been plotted in Fig. 1 for the ideal case $n=0$ (cavity at $0 \mathrm{~K}$ ). The function $K_{+}$takes its most forbidden values for $\Omega t$ equal to $n \pi / 3, n$ being any integer that is not a multiple of 3. The function $K_{-}$takes its first minimum at $\pi / 6$ and then shows a similar periodic behavior.

In a more realistic situation, when the cavity contains an indefinite number of photons, the Rabi solution (15) has to

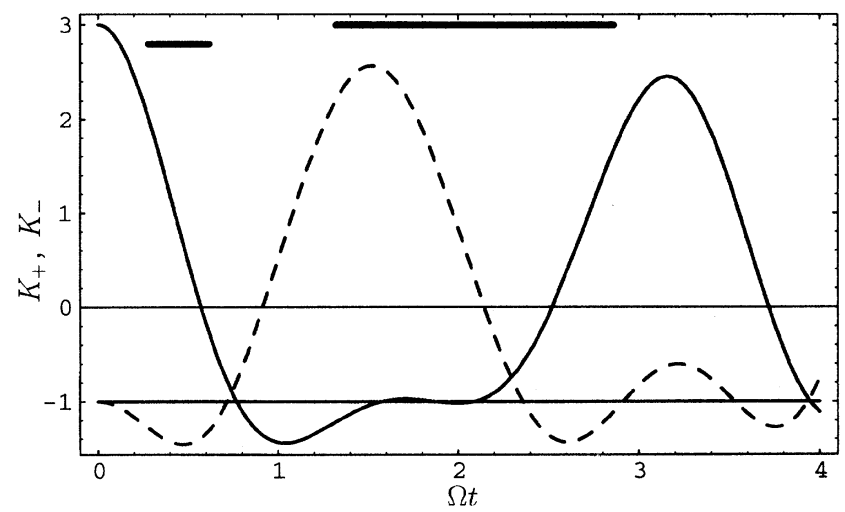

FIG. 3. $K_{ \pm}$versus $\Omega t$ at $T=0.5 \mathrm{~K}$. Violations of Leggett-Gargtype inequalities occur whenever either curve goes below -1 . The thick horizontal segments indicate the ranges of interaction times currently accessible for the two Rydberg transitions.

be averaged over the probability $p_{n}$ of having initially $n$ photons in the mode. Within the Jaynes-Cummings model that we are using [15],

$$
P_{e e}(t)=\sum_{n=0}^{\infty} p_{n} \cos ^{2}[\Omega \sqrt{n+1} t],
$$

and the corresponding autocorrelation now reads

$$
K_{Q}(t)=\sum_{n=0}^{\infty} p_{n} \cos [2 \Omega \sqrt{n+1} t] .
$$

The probabilities $p_{n}$ are easily calculated from Planck's blackbody formula corresponding to the temperature of the cavity (see below). The measurement of the autocorrelation function (16) requires an additional assumption, namely $P_{e g}=P_{g e}$. This condition is fulfilled in the JaynesCummings model, but it is also plausible in a realist theory on the basis of time reversal invariance. Hence, it is easy to see that (16) becomes

$$
K_{e x p}=P_{e e}-P_{e g},
$$

which gives the autocorrelation in terms of easily measurable quantities.

Consider a situation in which a beam of excited two-level atoms is injected into the cavity. By means of a Fizeau velocity selector it is possible to fix different atom-field interaction times $[12,13]$. The flux is kept low enough for at most one atom at a time to be inside the resonator. If we assume that, in all cases, the interaction time is much shorter than the cavity damping time $\tau$, the relaxation of the field mode can be ignored while an atom is inside the cavity and the description of the composite system is then straightforward [16]. In addition, for the proposed stationary regime to be applicable, the incoming flux must be kept low enough to ensure that the time interval between successive atoms is greater than $\tau$. Under these conditions, the state of the cavity is not affected [16] by the passage of successive atoms, so that each atom encounters a pure thermal field when it enters the cavity. Then 


$$
p_{n}=\frac{\bar{n}^{n}}{(\bar{n}+1)^{n+1}},
$$

where $\bar{n}$ is the temperature-dependent mean photon number. This would justify the term stationary cavity used above.

In order to analyze the behavior of the functions $K_{ \pm}(t)$ in this case, we will consider the specific example of a high- $Q$ resonator, which can be tuned to two different Rydberg transitions, $63 p_{3 / 2} \rightarrow 61 d_{3 / 2}(21506.5 \mathrm{MHz})$ and $63 p_{3 / 2} \rightarrow 61 d_{5 / 2}$ $(21456.0 \mathrm{MHz})$ of ${ }^{85} \mathrm{Rb}$, with an experimental setup analogous to that described in Refs. $[12,13]$. The corresponding Rabi frequencies are estimated to be 1.5 and $7 \mathrm{KHz}$, respectively. For a temperature of $3 \mathrm{~K}$, the quality factor of the cavity is $Q=6 \times 10^{7}$. Under these conditions, $\vec{n}=2.5$ and $\tau=500 \mu \mathrm{s}$. If the temperature is reduced to $0.5 \mathrm{~K}$ by means of a ${ }^{3} \mathrm{He}$ cryostat, $Q$ is increased to $3 \times 10^{10}$ and the storage time is now $0.2 \mathrm{~s}$ with a mean photon number reduced to 0.15 . In order to justify the stationarity assumption, the atomic flux cannot exceed the value of $2000 \mathrm{~s}^{-1}$ in the low- $Q$ domain, and it has to be reduced below $5 \mathrm{~s}^{-1}$ when the temperature is $0.5 \mathrm{~K}$. The behavior of $K_{ \pm}(t)$ has been plotted in Figs. 2 and 3 for these two different temperature regimes. The experimental data presently available cover the range between 30 and $130 \mu \mathrm{s}$ [12], which corresponds, for the two different transitions considered, to the thick segments plotted in the top. When $T=3 \mathrm{~K}$, only violations of the realist bound for $K_{-}$may be observed for the transition with smaller Rabi frequency; for instance, $K_{-}=-1.28$ for the pair of interaction times of 30 and $60 \mu \mathrm{s}$. However, with the statistics provided by Rempe et al. for $P_{e}(t)$, the extreme value of $K_{-}$has a range of uncertainty between -0.6 and -1.2 , so that no conclusive answer can be obtained. When the temperature is decreased to $0.5 \mathrm{~K}$, the departures from the realist bounds are enhanced, and in this case the behavior of $K_{ \pm}(t)$ differs only slightly from the situation in which the state of the cavity is described by a pure Fock state, plotted in Fig. 1. In this case, it would be possible to detect forbidden values for both $K_{ \pm}$with the transition with larger $\Omega$. In order to observe violations of the bounds for $K_{ \pm}$in wider regions, the interaction times should be either increased above $130 \mu \mathrm{s}$, which demands velocities below $180 \mathrm{~m} / \mathrm{s}$ or reduced below $30 \mu \mathrm{s}$, for which velocities above $800 \mathrm{~m} / \mathrm{s}$ are required. Given that a very low flux is required in the lowtemperature regime, it would not be necessary to employ laser-cooling techniques in the first case, as the initial thermal distribution provides $2 \%$ of the atoms with the required conditions. As far as rapid atoms is concerned, the initial distribution supplies $6 \%$ in the suitable range, and also this possibility seems experimentally feasible.

Finally, note that, in the experiment envisaged here, it is necessary to evaluate the autocorrelation (16) at two interaction times $t$ and $2 t$ in order to test the time dependence of the functions $K_{ \pm}$. For that, absolute measurements of the probabilities $P_{e e}$ and $P_{e g}$ are required. When both channeltrons have the same efficiency, a sequence of such measurements would simply provide a scaled curve that can be identified with the absolute one if the faithful sample assumption is made. However, this method is not applicable if the atomic detectors have different efficiencies. In this case the corresponding efficiencies should be determined by an independent procedure.

Summarizing, the proposed experiment provides a scenario suitable for showing discrepancies from realist predictions bounded by Leggett-Garg-type inequalities. We believe that this proposal would help to clarify the incompatibility between QM and a realist framework, as well as giving a test of QM outside the purely microscopical domain.

We acknowledge financial support from DGICYT, Project No. PB-92-0507 (Spain).
[1] A. Einstein, B. Podolsky, and N. Rosen, Phys. Rev. 47, 777 (1935).

[2] E. Schrödinger, Naturwis. 23, 807 (1935).

[3] J. S. Bell, Physica 1, 195 (1964); Rev. Mod. Phys. 38, 447 (1966).

[4] Quantum Mechanics versus Local Realism, edited by F. Selleri (Plenum, New York, 1988).

[5] N. D. Mermin, Rev. Mod. Phys. 65, 803 (1993).

[6] A. J. Leggett and A. Garg, Phys. Rev. Lett. 54, 857 (1985).

[7] C. D. Tesche, Phys. Rev. Lett. 64, 2358 (1990).

[8] J. P. Paz and G. Mahler, Phys. Rev. Lett. 71, 3235 (1993).

[9] S. J. D. Phoenix and S. M. Barnett, J. Mod. Opt. 40, 979 (1993).
[10] J. I. Cirac and P. Zoller, Phys. Rev. A 50, R2799 (1994).

[11] E. Santos, Phys. Rev. Lett. 66, 1388 (1991); 68, 2702 (1992); Phys. Rev. A 46, 3646 (1992).

[12] G. Rempe, H. Walther, and N. Klein, Phys. Rev. Lett. 58, 353 (1987).

[13] G. Rempe, F. Schmidt-Kaler, and H. Walther, Phys. Rev. Lett. 64, 2783 (1990).

[14] L. Hardy, D. Home E. J. Squires, and M. A. B. Whitaker, Phys. Rev. A 45, 4267 (1992).

[15] L. Allen and J. H. Eberly, Optical Resonance and Two Level Atoms (Wiley, New York, 1975), p. 159.

[16] P. Filipowicz, J. Javanainen, and P. Meystre, Phys. Rev. A 34, 3007 (1986). 